\title{
OPEN Fixation probabilities in network structured meta-populations
}

\author{
Sedigheh Yagoobi ${ }^{\bowtie}$ \& Arne Traulsen \\ The effect of population structure on evolutionary dynamics is a long-lasting research topic in \\ evolutionary ecology and population genetics. Evolutionary graph theory is a popular approach to \\ this problem, where individuals are located on the nodes of a network and can replace each other via \\ the links. We study the effect of complex network structure on the fixation probability, but instead \\ of networks of individuals, we model a network of sub-populations with a probability of migration \\ between them. We ask how the structure of such a meta-population and the rate of migration affect \\ the fixation probability. Many of the known results for networks of individuals carry over to meta- \\ populations, in particular for regular networks or low symmetric migration probabilities. However, \\ when patch sizes differ we find interesting deviations between structured meta-populations and \\ networks of individuals. For example, a two patch structure with unequal population size suppresses \\ selection for low migration probabilities.
}

Evolutionary graph theory ${ }^{1}$ analyses the evolutionary dynamics in network-structured populations, where individuals are located on the nodes of a graph and can replace each other via the links. This approach can be motivated by several biological systems, from the spread of cancerous mutations through colonic crypts to the invasion of ecosystems structured by rivers. One of the major goals of evolutionary graph theory ${ }^{1}$ is to assess the effect of underlying population structure on the fixation probability (the probability of ultimate fixation of a mutant $)^{2-6}$ and the fixation time ${ }^{7-10}$. An important aim is to find an optimized structure to speed up or slow down the spread of a newly arising mutant ${ }^{11-13}$.

However, to apply such models, a change in perspective is often necessary: In many biological applications, the nodes correspond to small populations and not to single individuals ${ }^{14-16}$. Replacement of individuals via the links is then exchanged with migration where the immigrant displaces one from the resident individuals. This leads to network-structured meta-populations, where a network is formed by individual populations connected via migration. An important question that arises in the application of evolutionary graph theory is thus whether the results derived for networks of individuals carry over to networks of small populations.

Traditionally, such meta-populations have been analyzed extensively in ecology, where they correspond to fragmented habitats ${ }^{17-19}$. The dynamics of the meta-population is driven by the exchange of individuals between subpopulations. Nevertheless, the focus of these studies is usually quite different, as they aim to address complex questions arising in ecology by asking for the impact of such population structure. Also in population genetics, the fixation probability in subdivided populations (meta-populations) has been investigated extensively, see e.g. the study by Maruyama in $1970^{20}$, who has shown that the fixation probability is not altered by the subdivision of a population into partially isolated patches under certain assumptions. Later, other types of structured populations and alternative modes of selection and evolutionary dynamics have been discussed in this context ${ }^{21-25}$. However, the focus in the context of population genetics is not on the network structure of the meta-population structure. Typically, in these systems individuals from every patch have the freedom to migrate to all the other patches, whereas in our system individuals migrate to a small subset of adjacent patches.

Also, evolutionary game dynamics and the evolution of cooperation have been studied in subdivided populations ${ }^{2,26-32}$. Yet, with few exceptions, see e.g. ${ }^{33}$, these studies assume a well-mixed population of patches.

Here, we extend the typical models of evolutionary graph theory to network-structured meta-populations. In contrast to previous work that heads into this direction, our focus is on very basic models of populations of fixed size and a small number of patches. We discuss limiting cases and show how they can be addressed with the available analytical tools. We show that the isothermal theorem holds for any meta-populations with the regular structure. Since an exact solution for the fixation probability is not feasible when the network structure is complicated, an approximation for small migration probability is used to compute the fixation probability. Finally, we discuss the two-patch meta-population and meta-star, where several leaf patches are connected only to one central patch, when the migration probability is high. 
a

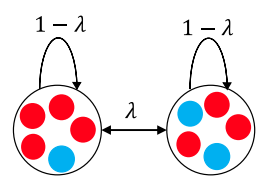

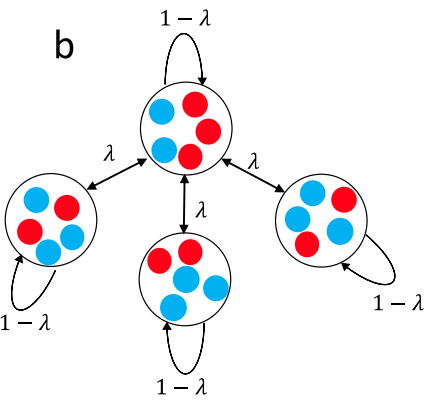

Figure 1. Network of populations; the population consists of well-mixed subpopulations coupled through migration (a) two-patch meta-population with local patch size $N_{1}=N_{2}=5$, (b) meta-star with $M=4$ patches and local population size $N / M=5$.

\section{Model}

We consider a finite population of size $N$ distributed in a network of $M$ patches (Fig. 1). The local population size of patch $j$ is constant and given by $N_{j}$. Each individual can reproduce either within its own patch or place its offspring into an adjacent patch.

We consider two types of individuals, mutant and wild-type. We start with a wild-type population of size $\sum_{j=1}^{M} N_{j}$. In the first step, a random wild-type individual turns into a mutant. We work with a standard Birth-death process with global selection for birth and random local death ${ }^{1,34}$ : In each time step, one individual is selected from the whole population at random, but proportional to its fitness, to produce identical offspring. Afterwards, the newborn replaces one of the individuals within its patch, including its mother, with probability $1-\lambda$, or it replaces one of the individuals in a random adjacent patch with probability $\lambda$. To be more precise, suppose that patch $i$ is connected to a set of other patches $V$. If $\alpha$ is an individual in patch $i$ and $\beta$ is an individual in patch $j \neq i$, then the probability that an offspring of $\alpha$ replaces $\beta$ is equal to $\frac{\lambda}{\sum_{k \in V} N_{k}}$, where $N_{k}$ is the population size in patch $k$. If $\alpha$ and $\beta$ are individuals in the same patch, $i$, the same probability is $\frac{1-\lambda}{N_{i}}$.

Our goal is to examine how likely this new mutant takes over the whole population and how this probability changes with the migration probability and the network structure.

For our model, the comparison with the fixation probability within a single patch of a well-mixed population is important. Therefore, we first recall how to calculate this quantity. Suppose the population of size $N$ consists of wild-types with fitness 1 only. If a new beneficial or deleterious mutant with fitness $r$ appears in the population, the evolution of the population will lead to the fixation or extinction of the mutant. As we adopt a Birth-death process the fixation probability, $\phi_{i}^{N}$, starting with $i$ mutants is determined by Refs. ${ }^{3-37}$

$$
\phi_{i}^{N}=T^{i+} \phi_{i+1}^{N}+T^{i-} \phi_{i-1}^{N}+\left(1-T^{i+}-T^{i-}\right) \phi_{i}^{N},
$$

where $T^{i+}=\frac{r i}{N-i+r i} \frac{N-i}{N}$ is the probability that the number of mutants increases from $i$ to $i+1$ and $T^{i-}=\frac{N-i}{N-i+r i} \frac{N-i+r i}{N}$ is the probability that the number of mutants decreases from $i$ to $i-1$. With this recursive relation and the boundary conditions $\phi_{0}^{N}=0$ and $\phi_{N}^{N}=1$, the fixation probability of a single mutant equals

$$
\phi_{1}^{N}(r)=\frac{1}{1+\sum_{k=1}^{N-1} \prod_{i=1}^{k} \frac{T^{i-}}{T^{i+}}}= \begin{cases}\frac{1-\frac{1}{r}}{1-\frac{1}{r^{N}}} & r \neq 1 \\ \frac{1}{N} & r=1 .\end{cases}
$$

This classical result will be an important reference point for our further considerations, we will denote it by $\phi_{\mathrm{wm}}^{N}(r)$ in the remainder (where "wm" stands for well-mixed).

\section{Results}

Regular structures and isothermal theorem. For networks where each node represents a single individual, the isothermal theorem of evolutionary graph theory shows that the fixation probability is the same as the fixation probability of a well-mixed population if the temperature distribution is homogeneous across the whole population ${ }^{1}$. The temperature of a node defined as the sum over all the weights leads to that node. This theorem extends to structured meta-populations for any migration probability $\lambda$ : If the underlying structure of the metapopulation that connects the patches is a regular network and the local population size is identical in each patch, the temperature of all individuals is identical, regardless of the value of the migration probability. Therefore, the fixation probability in a population with such a structure is the same as the fixation probability in a well-mixed population of the same total population size $N=\sum_{j=1}^{M} N_{j}$, given by $\phi_{\mathrm{wm}}^{N}(r)$.

Small migration regime. If the migration probability is small enough such that the time between two subsequent migration events $\left(\sim \frac{1}{\lambda}\right)$ is much longer than the absorption time within any patch, then at the time of each migration event we may suppose that the meta-population is in a homogeneous configuration ${ }^{22,28}$. In other words, the low migration regime is an approximation in which we neglect the probability that the metapopulation is not in a homogeneous configuration at the time of migration events. We define a homogeneous 
configuration of the meta-population as a configuration in which in all patches either all individuals are mutants, or all are wild-types.

Therefore, instead of having $2^{N}$ states, where $N$ is the population size, the system has only $2^{M}$ states, where $M$ is the number of patches. Thus, we can calculate the fixation probability exactly as in the case of a standard evolutionary graph model where each node represents a single individual but with a modified transition probabilities.

In a network with homogeneous patches, in order to increase the number of homogeneous mutant-patches one individual mutant needs to migrate to one of its neighbouring homogeneous wild-type-patches and reaches fixation there. For example if node $j$ is occupied by mutants and one of its neighbouring patches, node $k$, is occupied by wild-types, the probability that one mutant individual from patch $j$ migrates to patch $k$ and reaches fixation there is $\frac{\lambda}{\operatorname{deg}(\mathrm{j})} \phi_{\mathrm{wm}}^{N_{k}}(r)$, where $\operatorname{deg}(\mathrm{j})$ is the degree of node $j$ to take into account that the mutant can move to different patches. This is analogous to the probability that one mutant in node $j$ replaces one wild-type in node $k, T^{j \rightarrow k}$, in the network of individuals.

Similarly, if node $j$ is occupied by wild-types and one of its neighbouring patches, node $j$, is occupied by mutants the probability that one wild-type individual from patch $j$ migrates to patch $k$ and reaches fixation there equals to $\frac{\lambda}{\operatorname{deg}(\mathrm{j})} \phi_{\mathrm{wm}}^{N_{k}}(1 / r)$ where $\operatorname{deg}(\mathrm{j})$. Overall, we can move from network of individuals to the network of homogeneous patches by replacing the transition probabilities with the product of migration and fixation probabilities.

Two-patch meta-population. The simplest non-trivial case is the fixation probability in a two-patch meta-population with different local size for small migration probability $\lambda$. If the migration probability $\lambda$ is very small, a new mutant first needs to take over its own patch and only then the first migrant arrives in the second patch. To be more precise, the time between two migration events has to be much higher than the typical time that it takes for the migrant to take over the patch or go extinct again ${ }^{38}$. In this case, we can divide the dynamics into two phases: A first phase in which a mutant invades one patch and a second phase in which a homogeneous patch of mutants invades the whole meta-population. Assume a new mutation arises in patch 1 . Only if this mutant reaches fixation in patch 1 , it also has a chance to reach fixation in patch 2 . When patch 1 consists of only mutants and patch 2 consists of only wild-types, there are two possibilities for the ultimate fate of the mutant:

(i) Eventually, the offspring of one mutant selected from patch 1 for reproduction will migrate to patch 2 and reach fixation there. The wild-type goes extinct. This happens with probability $\frac{N_{1} r}{N_{1} r+N_{2}} \phi_{\mathrm{wm}}^{N_{2}}(r)$.

(ii) Eventually, the offspring of one wild-type selected from patch 2 for reproduction will migrate to patch 1 and the mutant goes extinct. This occurs with probability $\frac{N_{2}}{N_{1} r+N_{2}} \phi_{\mathrm{wm}}^{N_{1}}\left(\frac{1}{r}\right)$.

Therefore, the probability that a single mutant arising in patch 1 reaches fixation in the entire population is

$$
\phi_{\mathrm{wm}}^{N_{1}}(r) \frac{\frac{N_{1} r}{N_{1} r+N_{2}} \phi_{\mathrm{wm}}^{N_{2}}(r)}{\frac{N_{1} r}{N_{1} r+N_{2}} \phi_{\mathrm{wm}}^{N_{2}}(r)+\frac{N_{2}}{N_{1} r+N_{2}} \phi_{\mathrm{wm}}^{N_{1}}\left(\frac{1}{r}\right)}=\phi_{\mathrm{wm}}^{N_{1}}(r) \phi_{\mathrm{wm}}^{N_{2}}(r) \frac{1}{\phi_{\mathrm{wm}}^{N_{2}}(r)+\frac{N_{2}}{N_{1}} \frac{1}{r} \phi_{\mathrm{wm}}^{N_{1}\left(\frac{1}{r}\right)}} .
$$

Similarly the probability that a mutant arising in patch 2 takes over the whole population equals

$$
\phi_{\mathrm{wm}}^{N_{2}}(r) \phi_{\mathrm{wm}}^{N_{1}}(r) \frac{1}{\phi_{\mathrm{wm}}^{N_{1}}(r)+\frac{N_{1}}{N_{2}} \frac{1}{r} \phi_{\mathrm{wm}}^{N_{2}}\left(\frac{1}{r}\right)} .
$$

If we assume that the mutant arises in a patch with a probability proportional to the patch size, the average fixation probability $\phi_{\bullet}$. in a two patch population for small migration probability is the weighted sum of Eqs. (3a) and (3b),

$$
\begin{aligned}
\phi \bullet . & \phi_{\mathrm{wm}}^{N_{1}}(r) \phi_{\mathrm{wm}}^{N_{2}}(r) \\
& \times\left(\frac{\frac{N_{1}}{N_{1}+N_{2}}}{\phi_{\mathrm{wm}}^{N_{2}}(r)+\frac{N_{2}}{N_{1}} \frac{1}{r} \phi_{\mathrm{wm}}^{N_{1}}\left(\frac{1}{r}\right)}+\frac{\frac{N_{2}}{N_{1}+N_{2}}}{\phi_{\mathrm{wm}}^{N_{1}}(r)+\frac{N_{1}}{N_{2}} \frac{1}{r} \phi_{\mathrm{wm}}^{N_{2}\left(\frac{1}{r}\right)}}\right) .
\end{aligned}
$$

In the case of neutrality, $r=1$, we recover $\phi_{\bullet \bullet}=\frac{1}{N_{1}+N_{2}}$-the fixation probability in a population of the total size of the two patches. For identical patch sizes, $N_{1} \stackrel{N_{1}}{=} N_{2}$, Eq. (4) simplifies to

$$
\phi_{\bullet \bullet}=\left(\phi_{\mathrm{wm}}^{N_{1}}(r)\right)^{2} \frac{1}{\phi_{\mathrm{wm}}^{N_{1}}(r)+\frac{1}{r} \phi_{\mathrm{wm}}^{N_{1}}\left(\frac{1}{r}\right)}=\phi_{\mathrm{wm}}^{2 N_{1}}(r),
$$

where the simplification to the fixation probability within a single population of size $2 N_{1}$ reflects the validity of the isothermal theorem.

For $N_{1} \neq N_{2}$, we approximate Eq. (4) for weak and strong selection. Let us first consider highly advantageous mutants, $r \gg 1$. In this case, we have $\phi_{\mathrm{wm}}^{N_{1}}(r) \gg \phi_{\mathrm{wm}}^{N_{1}}\left(\frac{1}{r}\right)$ and thus we can neglect the possibility that a wild-type takes over a mutant patch if patch sizes are sufficiently large. The probability $\phi_{\bullet}$ then becomes a weighted average reflecting patch sizes. For identical patch size $N_{1}=N_{2}=N / 2$, it reduces to $\phi_{\bullet \bullet} \approx \phi_{\mathrm{wm}}^{N_{1}}(r)=\phi_{\mathrm{wm}}^{N / 2}(r)$. In other words, taking over the first patch is sufficient to make fixation in the entire population certain. For patches of very different size, $N_{1} \gg N_{2}$, we have $N \approx N_{1}$ and find $\phi_{\bullet .} \approx \phi_{\mathrm{wm}}^{N}(r)$, which implies that fixation is driven by the fixation process in the larger patch, regardless of where the mutant arises. Note that there is a difference between the case of identical patch size and very different patch size. The case of highly disadvantageous mutants, $r \ll 1$, can be handled in a very similar way. 


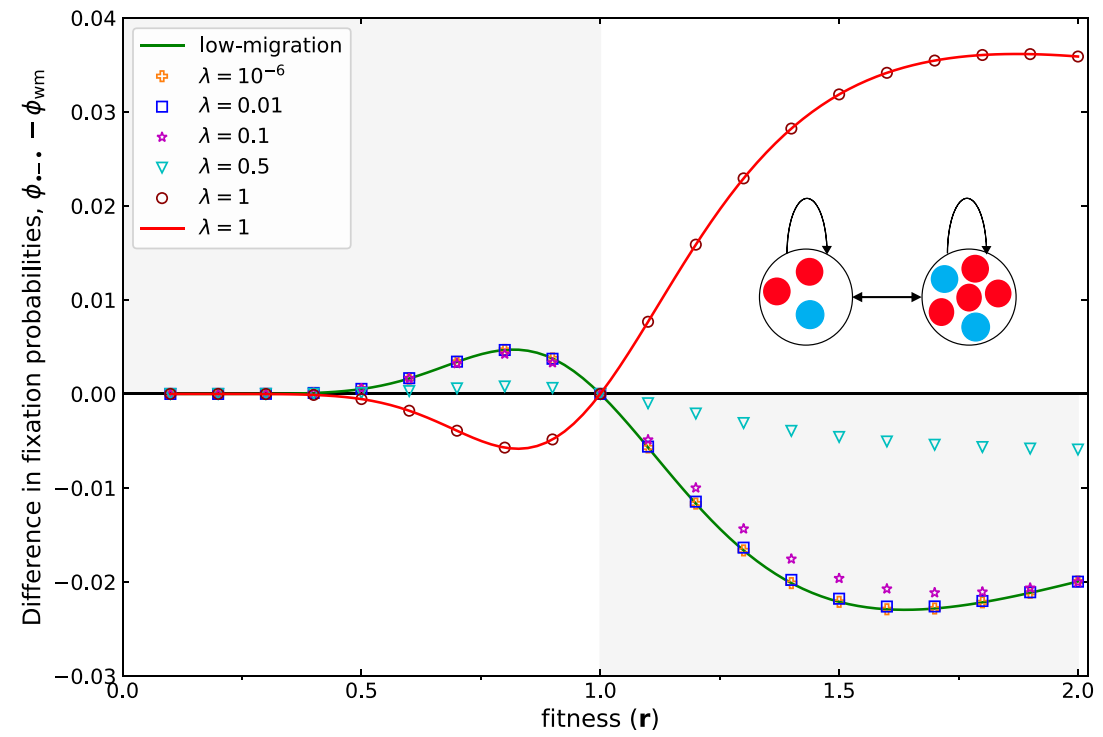

Figure 2. The difference between the fixation probability of a two-patch meta-population and a well-mixed population of the same size, $N=10$. The two patch sizes are $N_{1}=3$ and $N_{2}=7$. Lines show analytical results for low migration probabilities (Eq. 4) and migration probability $\lambda=1$ (Eq. 19) as a function of fitness. Symbols show numerical results based on a transition matrix approach ${ }^{39}$. The numerical result and analytical result for low migration probability and high migration match perfectly. In the low migration regime the two-patch metapopulation is a suppressor of selection, indicated by the fact that the symbols are never in the area with a white background. However, in the high migration regime $(\lambda=1)$, where simulations and analytical results again match, the two-patch meta-population is an amplifier of selection. The fixation probability for $\lambda=1$ is obtained analytically using the Martingale approach discussed by Monk ${ }^{41}$.

Next, we consider weak selection, $r \approx 1$. We can approximate the fixation probability as $\phi_{\mathrm{wm}}^{N}\left(r^{ \pm 1}\right) \approx \frac{1}{N} \pm \frac{N-1}{2 N}(r-1)$. With this, we find

$$
\phi_{\bullet} \approx \frac{1}{N_{1}+N_{2}}+\frac{1}{2}\left(1-\frac{1}{N_{1}+N_{2}}-\frac{\left(N_{1}-N_{2}\right)^{2}}{\left(N_{1}^{2}+N_{2}^{2}\right)^{2}} N_{1} N_{2}\right)(r-1) .
$$

For identical patch size $N_{1}=N_{2}=N / 2$, this reduces to

$$
\phi_{\bullet \bullet} \approx \frac{1}{N}+\frac{N-1}{2 N}(r-1)
$$

which is the known result for a single population of size $N=N_{1}+N_{2}$. When patches have very different size, $N_{1} \gg N_{2}$ such that $N \approx N_{1}$, we recover the same result. Thus, the difference between the fixation probability of a two-patch meta-population with identical patch size and the fixation probability of a two-patch metapopulation with very different patch size that we found for highly advantageous mutants is no longer observed for weak selection.

When migration probabilities become larger, our approximation is no longer valid and we need to rely on numerical approaches. Figure 2 illustrates the difference between the fixation probability of a two-patch structure meta-population and the equivalent well-mixed population of size $N_{1}+N_{2}$ when migration is low using Eq. (4) and comparing with the numerical approach in Ref. ${ }^{39}$.

While the fixation probability of the two-patch meta-population is very close to the fixation probability of the well-mixed population ${ }^{40}$, a close inspection reveals an interesting property: For low migration probabilities and $N_{1} \neq N_{2}$, the two patch structure is a suppressor of selection in the original sense of Lieberman et al. ${ }^{1}$ : For advantageous mutations, $r>1$, it decreases the fixation probability, whereas for disadvantageous mutations, $r<1$, it increases the fixation probability compared to the well mixed case. For weak selection, we show this analytically: For $r \approx 1$, we can write

$$
\begin{aligned}
& \phi_{\mathrm{wm}}^{N_{1}+N_{2}} \approx \frac{1}{N_{1}+N_{2}}+\frac{N_{1}+N_{2}-1}{2\left(N_{1}+N_{2}\right)}(r-1) \\
& \phi_{\bullet \bullet} \approx \phi_{\mathrm{wm}}^{N_{1}+N_{2}}-N_{1} N_{2} \frac{\left(N_{1}-N_{2}\right)^{2}}{2\left(N_{1}^{2}+N_{2}^{2}\right)^{2}}(r-1) .
\end{aligned}
$$

While the difference to the well mixed case vanishes for $N_{1}=N_{2}$ in first order in $r-1$, the fixation probability of the two patch structure is larger for $r<1$ and smaller for $r>1$. Thus, under weak selection the two patch structure with $N_{1} \neq N_{2}$ is a suppressor of selection. 
While the structure remains a suppressor of selection for most values of the migration probability $\lambda$, Fig. 2 reveals that for very large $\lambda$ it becomes an amplifier of selection.

The meta-star. Here, we approximate the fixation probability $\phi_{\star}$ of a meta-star with $M-1$ leaves for low migration probability. For simplicity, we assume that all patches are of the same size $N_{1}=\frac{N}{M}$ and omit the notation for patch size. As long as the migration probability is sufficiently low, such that before the next migration the immigrant gets fixed or lost, patches tend to be homogeneous. We denote the number of homogeneous mutant patches among the leaves by $j$ and use a lower index to represent the state of the central patch, which is either occupied by wild-types (o) or by mutants $(\bullet)$. The number of homogeneous mutant patches increases in two ways:

(i) the center is occupied by mutants and is selected for birth and its offspring migrates to one of the peripheral homogeneous wild-type patches and reaches fixation in that patch,

$$
T_{\bullet \rightarrow \bullet}^{j+}=\frac{r}{(j+1) r+M-1-j} \frac{M-1-j}{M-1} \lambda \phi_{\mathrm{wm}}^{N_{1}}(r)
$$

where $\phi_{\mathrm{wm}}^{N_{1}}(r)$ determines the fixation probability of a mutant in a local population,

(ii) the center is occupied by wild-types and one of the homogeneous mutant leaves is selected for birth and its offspring migrates to the center and gets fixed there,

$$
T_{\circ \rightarrow \bullet}^{j}=\frac{j r}{j r+M-j} \lambda \phi_{\mathrm{wm}}^{N_{1}}(r) .
$$

Note that the number of homogeneous mutant leave nodes cannot increase if the center is occupied by wildtype individuals, i.e. $T_{\circ \rightarrow \circ}^{j+}=0$. Similarly, the number of homogeneous mutant leave nodes cannot decrease if the center is occupied by mutants, i.e. $T_{\bullet \rightarrow \bullet}^{j-}=0$. Thus, the number of homogeneous mutant patches can decrease in two ways,

(i) the center is occupied by wild-types and is selected for birth and its offspring migrates to one of the homogeneous mutant leaves and gets fixed there,

$$
T_{\circ \rightarrow \circ}^{j-}=\frac{1}{j r+M-j} \frac{j}{M-1} \lambda \phi_{\mathrm{wm}}^{N_{1}}\left(\frac{1}{r}\right)
$$

where $\phi_{\mathrm{wm}}^{N_{1}}\left(\frac{1}{r}\right)$ is the fixation probability of a wild-type in a local mutant population, or

(ii) the center is occupied by mutants and one of the leaves is selected for birth and its offspring migrates to the center and gets fixed there,

$$
T_{\bullet \rightarrow \circ}^{j}=\frac{M-1-j}{(j+1) r+M-1-j} \lambda \phi_{\mathrm{wm}}^{N_{1}}\left(\frac{1}{r}\right) .
$$

For the star graph, depending on where the mutant emerges, two different fixation probabilities are defined: The fixation probability when a single mutant emerges in the center $\phi_{\bullet}^{0}$ and the fixation probability when a single mutant emerges in one of the leaves, $\phi_{\circ}^{1}$. Following the same arguments as in Ref. ${ }^{42}$, we find

$$
\begin{gathered}
\phi_{\bullet}^{0}=\frac{T_{\bullet}^{0}}{1+\left(1-T_{\bullet}^{0}\right) \sum_{j=1}^{M-2}\left(\frac{T_{\circ}^{j}}{T_{\bullet}^{j}}\right)^{j}} \\
\phi_{\circ}^{1}=\frac{1-T_{\circ}^{j}}{T_{\bullet}^{j}} \phi_{\bullet}^{0},
\end{gathered}
$$

where the probability to leave the state with a wild-type patch in the center and $j$ mutant patch leaves is

$$
T_{\circ}^{j}=\frac{T_{\circ \rightarrow \circ}^{j-}}{T_{\circ \rightarrow \bullet}^{j}+T_{\circ \rightarrow \circ}^{j-}}=\frac{\frac{1}{r} \phi_{\mathrm{wm}}^{N_{1}}\left(\frac{1}{r}\right)}{\frac{1}{r} \phi_{\mathrm{wm}}^{N_{1}}\left(\frac{1}{r}\right)+(M-1) \phi_{\mathrm{wm}}^{N_{1}}(r)},
$$

and the probability to leave the state with a mutant patch in the center and $j$ mutant patch leaves is

$$
T_{\bullet}^{j}=\frac{T_{\bullet \rightarrow \bullet}^{j+}}{T_{\bullet \rightarrow \bullet}^{j+}+T_{\bullet \rightarrow \circ}^{j}}=\frac{r \phi_{\mathrm{wm}}^{N_{1}}(r)}{r \phi_{\mathrm{wm}}^{N_{1}}(r)+(M-1) \phi_{\mathrm{wm}}^{N_{1}\left(\frac{1}{r}\right)}} .
$$

Note that the two probabilities $T_{\circ}^{j}$ and $T_{\bullet}^{j}$ are independent of $j$ in our particular case. Thus, also their ratio $\Gamma=T_{\circ}^{j} / T_{\bullet}^{j}$ is independent of $j$, which makes our calculation of $\phi_{\bullet}^{0}$ easier. 


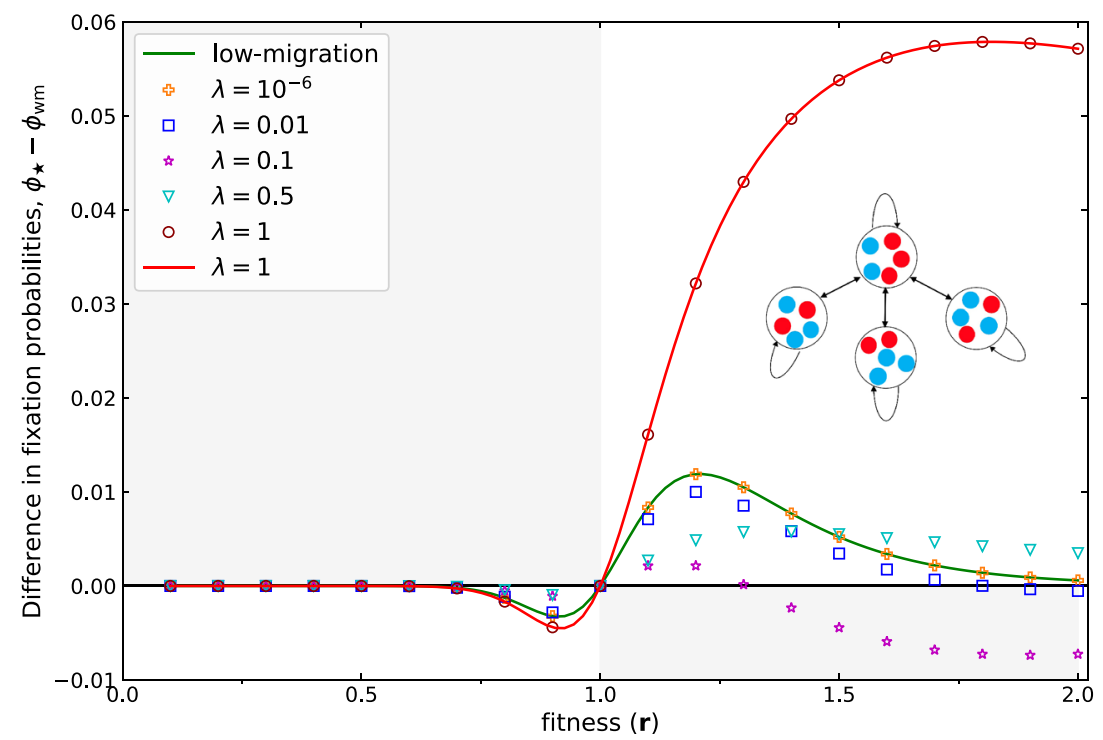

Figure 3. The difference between the fixation probability of a meta-star and the equivalent well-mixed population of size with $M=4$ patches and identical local size in each patch $N_{1}=N / M=5$ for different fitness values. Lines show analytical results for low migration probabilities (Eq. 18) and migration probability $\lambda=1$ (Eq. 19) symbols show numerical results based on a transition matrix approach ${ }^{39}$ for $\lambda=10^{-6}, \lambda=0.01$, $\lambda=0.1$, and $\lambda=0.5$. For migration probability $\lambda=10^{-6}$, we observe an almost perfect agreement, the low migration result serves as a good approximation. The fixation probability in $\lambda=1$ is obtained analytically using the Martingale approach. For extremely high and low migration probability the meta-star acts as an amplifier of selection (such that the lines only pass through the white shaded area of the plot) while in the intermediate migration regime shows a very different behavior where it could be an amplifier or a suppressor of selection depending on the fitness value.

Evaluating Eq. (14), we find the average fixation probability in the entire patch structured meta-population starting from a single homogeneous mutant patch,

$$
\phi_{\text {patch }}=\frac{M-1}{M} \phi_{\circ}^{1}+\frac{1}{M} \phi_{\bullet}^{0} .
$$

Therefore, the fixation probability of the whole population equals

$$
\phi \star=\phi_{\mathrm{wm}}^{N_{1}}(r) \phi_{\text {patch }} \text {. }
$$

Figure 3 illustrates the fixation probability of a meta-star as a function of fitness. Numerical solutions for low migration agree very well with the low migration approximation. According to this plot, the meta-star is an amplifier of selection in the low migration regime-similar to the star network of individuals ${ }^{1}$. A numerical investigation of Eq. (18) reveals that this result carries over to larger $N_{1}$ as well. For any value of $M$ and $N_{1}$ between 1 and 100, we find that the star network of patches amplifies selection. However, as expected from earlier work ${ }^{40}$, the extent of amplification becomes smaller with growing population size.

Using the same approach as we used for the two patch meta-population, we find that the meta-star is an amplifier for small and high migration probability, but not in between. For intermediate migration probability, it is only a piecewise amplifier ${ }^{43,44}$ and does not fall into one of the originally defined categories, see Fig. 3.

The meta-star in low migration is equivalent to the "star of islands" discussed by Allen et al. ${ }^{45}$. In their study for death-Birth updating they found that the comparison of the size of the hub to the size of the leaves makes a determinative difference. When the leaves are larger, the structure amplifies under weak selection; when the hub is larger, it suppresses under weak selection. When the hub and leaves are the same size, the structure acts as a "reducer", meaning that it lessens the fixation probability for all $r$ not equal to 1 (termed "suppressor of fixation" elsewhere $\left.{ }^{34}\right)$. Doing the same comparison in the whole range of selection, we find that the meta-star under Birth-death is an amplifier when the hub has greater or equal size to the leaves, and a transient amplifier when the leaves are larger than the hub.

High migration probability. For moderate migration probabilities, it is challenging to calculate the fixation probability. However, in the case of the maximum possible migration probability, $\lambda=1$, the two-patch meta-population and meta-star transform to complete bipartite graphs: In the two-patch meta-population, every offspring will be immediately moved to the other patch. In the meta-star, the offspring of individuals in the center node will be placed in a random leaf, whereas the offspring of the individuals in the leaf nodes will be placed in the center. Thus, the meta-star can be thought of as a bipartite graph in which one part is made out of all leaf nodes and the other part out of the center. 
The fixation probability of complete bipartite graphs has been calculated analytically previously ${ }^{41,44,46}$ using the specific features of Martingales. The probability to reach fixation if the initial mutant arises either in patch $1, \phi_{\mathrm{bp}}^{1}$ or patch $2, \phi_{\mathrm{bp}}^{2}$ are

$$
\phi_{\mathrm{bp}}^{1}=\frac{h_{1}-1}{\left(h_{1}\right)^{N_{1}}\left(h_{2}\right)^{N_{2}}-1}, \quad \phi_{\mathrm{bp}}^{2}=\frac{h_{2}-1}{\left(h_{1}\right)^{N_{1}}\left(h_{2}\right)^{N_{2}}-1},
$$

where $h_{1}=\frac{\frac{N_{2}}{N_{1}}+\frac{1}{r}}{\frac{N_{2}}{N_{1}}+r}$ and $h_{2}=\frac{\frac{N_{1}}{N_{2}}+\frac{1}{r}}{\frac{N_{1}}{N_{2}}+r}$. The average fixation probability is

$$
\phi_{\mathrm{bp}}=\frac{N_{1} \phi_{\mathrm{bp}}^{1}+N_{2} \phi_{\mathrm{bp}}^{2}}{N_{1}+N_{2}} .
$$

In the case where one of the patch size is much larger than the other, $N_{2} \gg N_{1}$, the fixation probability converges to the fixation probability of a star graph $\frac{1-1 / r^{2}}{1-1 / r^{2 N_{2}}}$.

As discussed above, a star-structured meta-population in $\lambda=1$ can be reduced to a complete bipartite graph. As a result the fixation probability of a star meta-population with $M-1$ leaves and population size $N$ such that the population distributing homogeneously in all the patches is obtained by replacing $N_{1}$ with $\frac{(M-1) N}{M}$ and $N_{2}$ with $\frac{N}{M}$ in Eqs. (19) and (20).

As shown in Figs. 2 and 3 both the two-patch meta-population and meta-star are amplifiers of selection for $\lambda=1$. It has been proven in Ref. ${ }^{47}$ that a complete bipartite amplifies selection for weak selection. This can also be seen from a Taylor expansion of the difference between Eq. (20) and the corresponding result for the well mixed population at $r=1$, which leads to

$$
\phi_{\mathrm{bp}}-\phi_{\mathrm{wm}} \approx \frac{1}{2} \frac{\left(N_{1}-N_{2}\right)^{2}}{\left(N_{1}+N_{2}\right)^{2}} \frac{N_{1}\left(N_{1}-1\right)+N_{2}\left(N_{2}-1\right)}{N_{1}^{2}+N_{2}^{2}}(r-1) .
$$

This quantity is positive for $r>1$ and negative for $r<1$, such that the structure is an amplifier of selection for weak selection. Eq. (20) reveals this fact holds for the whole range of selection strength. If we have a fixed population of size $N$ on a complete bipartite graph, for fitness values $r>1$ the minimum fixation probability occurs when the two patch sizes are identical, $N_{1}=N_{2}=N / 2$. Similarly, for fitness values $r<1$ the maximum fixation probability occurs when the two patch sizes are identical, $N_{1}=N_{2}=N / 2$.

Since a complete bipartite graph with identical patch size is an isothermal graph and its fixation probability is the same as the fixation probability of a well-mixed population, we conclude that any complete bipartite graph is the amplifier of selection when $N_{1} \neq N_{2}$. This result is implicitly contained in Refs. ${ }^{41,46}$, but deserves special attention: It implies that a graph can be turned into the amplifier if we enforce a very large degree of exchange of individuals between patches. Combined with the observation that many graphs of individuals are amplifiers of selection ${ }^{34}$, it suggests that it may be easier to construct amplifiers of selection than suppressors of selection in undirected networks ${ }^{5,48}$.

\section{Discussion}

Here, we have extended evolutionary graph theory from graphs of individuals to evolutionary graph theory of populations. We have investigated how the structure and migration probability influence the probability to fixation. For regular networks and patches of identical size, any evolutionary graph of populations has the same fixation probability as the well mixed population for any migration probability-a result that follows directly from the isothermal theorem ${ }^{1}$.

However, for non-regular networks or patches of different size, this is no longer the case and the dynamics depends on the migration probability. If the migration probability is small enough, such that the time to fixation in one patch is small compared to the time between two subsequent migration events, we can use time-scale separation to approximate the fixation probability. Using this approximation, we show that the two-patch metapopulation suppresses selection whenever the two patches have different size. Based on the same approximation, we have shown that the meta-star amplifies selection. In the high migration regime, the two-patch metapopulation and the meta-star can be viewed as complete bipartite graphs. Evolutionary dynamics in bipartite graphs has been studied in Refs. ${ }^{41,46}$. Both the two-patch meta-population and the meta-star are amplifiers of selection in this high migration regime.

Here, in order to be as close to the original ideas of evolutionary graph theory and the popular Birth-death updating as possible, we have focussed on meta-populations in which selection is global. Thus, individuals compete across the entire meta-population for reproduction. Another possible condition would be when the competition is local i.e. among the individuals belonging to the same patch. This would correspond to a process where a site becomes available and there is local competition to fill it, similar to death-Birth dynamics in networks of individuals ${ }^{45,49}$. The choice of this dynamics can have massive consequences on the amplification or suppression properties of a graph ${ }^{34}$. In general, structured meta-populations allow many additional selection processes that are still to be analyzed.

\section{Methods}

Depending on the network and the range of migration we adopt three different approaches to calculate the fixation probability: 
(i) In the very low migration probability we implement an analytical approximation for the fixation probability using the time scale separation between migration and fixation in a single patch.

(ii) For intermediate migration probability we compute the fixation probability numerically using the transition matrix based on approach published in Ref. ${ }^{39}$. The entries of the transition matrix represent the transition probability between different possible states. The number of states depend upon the symmetry of the network.

(iii) For very high migration probability we calculate the fixation probability analytically using the Martingales introduced in Refs. ${ }^{46,50}$.

We model the evolutionary dynamics using the Moran process. The code to reproduce our figures is available at https://github.com/s-yagoobi/fixation-probability.

Received: 16 April 2021; Accepted: 23 August 2021

Published online: 09 September 2021

\section{References}

1. Lieberman, E., Hauert, C. \& Nowak, M. A. Evolutionary dynamics on graphs. Nature 433, 312-316 (2005).

2. Allen, B. et al. Evolutionary dynamics on any population structure. Nature 544, 227-230 (2017).

3. Nowak, M. A., Tarnita, C. E. \& Antal, T. Evolutionary dynamics in structured populations. Philos. Trans. R. Soc. B 365, 19-30 (2010).

4. Broom, M., Rychtář, J. \& Stadler, B. T. Evolutionary dynamics on graphs-The effect of graph structure and initial placement on mutant spread. J. Stat. Theory Pract. 5, 369-381 (2011).

5. Alcalde Cuesta, F., González Sequeiros, P. \& Lozano Rojo, Á. Suppressors of selection. PLoS One 12, e0180549 (2017).

6. Kuo, Y. P., Nombela-Arrieta, C. \& Carja, O. A theory of evolutionary dynamics on any complex spatial structure. bioRxiv. https:// doi.org/10.1101/2021.02.07.430151 (2021).

7. Hindersin, L. \& Traulsen, A. Counterintuitive properties of the fixation time in network-structured populations. J. R. Soc. Interface 11, 20140606 (2014).

8. Frean, M., Rainey, P. \& Traulsen, A. The effect of population structure on the rate of evolution. Proc. R. Soc. B 280, 20130211 (2013).

9. Ottino-Löffler, B., Scott, J. G. \& Strogatz, S. H. Takeover times for a simple model of network infection. Phys. Rev. E 96, 012313 (2017).

10. Hathcock, D. \& Strogatz, S. H. Fitness dependence of the fixation-time distribution for evolutionary dynamics on graphs. Phys. Rev. E 100, 012408 (2019).

11. Moeller, M., Hindersin, L. \& Traulsen, A. Exploring and mapping the universe of evolutionary graphs identifies structural properties affecting fixation probability and time. Commun. Biol. 2, 137 (2019).

12. Tkadlec, J., Pavlogiannis, A., Chatterjee, K. \& Nowak, M. A. Population structure determines the tradeoff between fixation probability and fixation time. Commun. Biol. 2, 1-8 (2019).

13. Tkadlec, J., Pavlogiannis, A., Chatterjee, K. \& Nowak, M. A. Fast and strong amplifiers of natural selection. Nat. Commun. 12, 4009. https://doi.org/10.1038/s41467-021-24271-w (2021).

14. Chakraborty, P. P., Nemzer, L. R. \& Kassen, R. Experimental evidence that metapopulation structure can accelerate adaptive evolution. bioRxiv. https://doi.org/10.1101/2021.07.13.452242 (2021).

15. Marrec, L., Lamberti, I., \& Bitbol A-F. Toward a universal model for spatially structured populations. arXiv preprint arXiv:2012. 06913. https://doi.org/10.1101/2020.12.12.422518 (2020).

16. Houchmandzadeh, B. \& Vallade, M. The fixation probability of a beneficial mutation in a geographically structured population. New J. Phy. 13(7), 073020 (2011).

17. Hanski, I. Metapopulation dynamics. Nature 396(6706), 41-49. https://doi.org/10.1038/23876 (1998) (Publisher: Nature Publishing Group).

18. Thrall, P. H. et al. Rapid genetic change underpins antagonistic coevolution in a natural host-pathogen metapopulation. Ecol. Lett. 15, 425-435 (2012).

19. Eriksson, A., Elías-Wolff, F. \& Mehlig, B. Metapopulation dynamics on the brink of extinction. Theor. Popul. Biol. 83, 101-122. https://doi.org/10.1016/j.tpb.2012.08.001 (2013).

20. Maruyama, T. On the fixation probability of mutant genes in a subdivided population. Genet. Res. 15, 221-225 (1970).

21. Barton, N. H. The probability of fixation of a favoured allele in a subdivided population. Genet. Res. 62, 149-157 (1993).

22. Tachida, H. \& Iizuka, M. Fixation probability in spatially changing environments. Genet. Res. 58, 243-251 (1991).

23. Maruyama, T. A simple proof that certain quantities are independent of the geographical structure of population. Theor. Popul. Biol. 5, 148-154 (1974).

24. Whitlock, M. C. Fixation probability and time in subdivided populations. Genetics 164, 767-779 (2003).

25. Cherry, J. L. \& Wakeley, J. A diffusion approximation for selection and drift in a subdivided population. Genetics 163, 421-428 (2003).

26. Eshel, I. On the neighbor effect and the evolution of altruistic traits. Theor. Popul. Biol. 3, 258-277 (1972).

27. Traulsen, A. \& Nowak, M. A. Evolution of cooperation by multilevel selection. Proc. Natl. Acad. Sci. 103, 10952-10955 (2006).

28. Hauert, C., Chen, Y.-T. \& Imhof, L. A. Fixation times in deme structured, finite populations with rare migration. J. Stat. Phys. 156, 739-759 (2014).

29. Hauert, C. \& Imhof, L. Evolutionary games in deme structured, finite populations. J. Theor. Biol. 299, 106-112 (2012).

30. Luo, S. A unifying framework reveals key properties of multilevel selection. J. Theor. Biol. 341, 41-52. https://doi.org/10.1016/j. jtbi.2013.09.024 (2014).

31. Cooney, D. B. The replicator dynamics for multilevel selection in evolutionary games. J. Math. Biol. 79, 101-154. https://doi.org/ 10.1007/s00285-019-01352-5 (2019).

32. Korolev, K. \& Nelson, D. R. Competition and cooperation in one-dimensional stepping-stone models. Phys. Rev. Lett. 107, 088103 (2011).

33. Akdeniz, A. \& van Veelen, M. The cancellation effect at the group level. Evolution 74, 1246-1254 (2020).

34. Hindersin, L. \& Traulsen, A. Most undirected random graphs are amplifiers of selection for Birth-death dynamics, but suppressors of selection for death-Birth dynamics. PLoS Comput. Biol. 11, e1004437 (2015).

35. Karlin, S. \& Taylor, H. M. A. A First Course in Stochastic Processes 2nd edn. (Academic, 1975).

36. Nowak, M. A. Evolutionary Dynamics: Exploring the Equations of Life (Harvard University Press, 2006).

37. Traulsen, A. \& Hauert, C. Stochastic evolutionary game dynamics. In Reviews of Nonlinear Dynamics and Complexity Vol. II (ed. Schuster, H. G.) 25-61 (Wiley-VCH, 2009).

38. Wu, B., Gokhale, C. S., Wang, L. \& Traulsen, A. How small are small mutation rates?. J. Math. Biol. 64, 803-827 (2012). 
39. Hindersin, L., Moeller, M., Traulsen, A. \& Bauer, B. Exact numerical calculation of fixation probability and time on graphs. Biosystems 150, 87-91 (2016).

40. Adlam, B. \& Nowak, M. A. Universality of fixation probabilities in randomly structured populations. Sci. Rep. 4, 1-6 (2014).

41. Monk, T., Green, P. \& Paulin, M. Martingales and fixation probabilities of evolutionary graphs. Proc. R. Soc. A 470, 20130730 (2014).

42. Broom, M. \& Rychtár, J. An analysis of the fixation probability of a mutant on special classes of non-directed graphs. Proc. R. Soc. A 464, 2609-2627 (2008).

43. Voorhees, B. Birth-death fixation probabilities for structured populations. Proc. R. Soc. A 469, 20120248 (2013).

44. Voorhees, B. \& Murray, A. Fixation probabilities for simple digraphs. Proc. R. Soc. A 469, 20120676 (2013).

45. Allen, B. et al. Transient amplifiers of selection and reducers of fixation for death-birth updating on graphs. PLoS Comput. Biol. 16, e1007529 (2020).

46. Monk, T. Martingales and the fixation probability of high-dimensional evolutionary graphs. J. Theor. Biol. 451, 10-18 (2018).

47. Allen, B. et al. Fixation probabilities in graph-structured populations under weak selection. PLoS Comput. Biol. 17, e1008695 (2021).

48. Pavlogiannis, A., Tkadlec, J., Chatterjee, K. \& Nowak, M. A. Construction of arbitrarily strong amplifiers of natural selection using evolutionary graph theory. Commun. Biol. 1, 1-8 (2018).

49. Tkadlec, J., Pavlogiannis, A., Chatterjee, K. \& Nowak, M. A. Limits on amplifiers of natural selection under death-birth updating. PLoS Comput. Biol. 16, e1007494 (2020).

50. Monk, T. \& van Schaik, A. Walds martingale and the conditional distributions of absorption time in the moran process. Proc. $R$. Soc. A 476, 20200135 (2020).

\section{Acknowledgements}

We thank Stefano Giaimo and Nikhil Sharma and Florence Bansept for constructive discussions on this subject.

\section{Author contributions}

A.T. designed the study. S.Y. designed and implemented the code and performed the numerical analysis. S.Y. and A.T., analyzed the simulation data and developed analytical calculations. Both authors wrote the manuscript together.

\section{Funding}

Open Access funding enabled and organized by Projekt DEAL.

\section{Competing interests}

The authors declare no competing interests.

\section{Additional information}

Correspondence and requests for materials should be addressed to S.Y.

Reprints and permissions information is available at www.nature.com/reprints.

Publisher's note Springer Nature remains neutral with regard to jurisdictional claims in published maps and institutional affiliations.

Open Access This article is licensed under a Creative Commons Attribution 4.0 International License, which permits use, sharing, adaptation, distribution and reproduction in any medium or format, as long as you give appropriate credit to the original author(s) and the source, provide a link to the Creative Commons licence, and indicate if changes were made. The images or other third party material in this article are included in the article's Creative Commons licence, unless indicated otherwise in a credit line to the material. If material is not included in the article's Creative Commons licence and your intended use is not permitted by statutory regulation or exceeds the permitted use, you will need to obtain permission directly from the copyright holder. To view a copy of this licence, visit http://creativecommons.org/licenses/by/4.0/.

(c) The Author(s) 2021 